\section{Matrix decomposition: An APL function to compute the Cholesky factor of a gramian matrix}

\author{
WILLIAM T. MCGOWAN III \\ Data Center, School of Medicine, University of South Carolina \\ Columbia, South Carolina 29208
}

This paper describes an APL function that computes the Cholesky factor of a gramian matrix. Cholesky, or square-root factorization, has been shown to be an accurate and efficient method of decomposing any symmetric positive definite (PD) matrix (e.g., most sum. of-cross-products and variance-covariance matrices) or positive semidefinite (PSD) into triangular form (Finn, 1974). The significance of this factorization procedure to statistical methods has long been recognized, and accordingly, computer programs (within a batch environment) have been developed for its computation (Bock \& Repp, Note 1; Finn, Note 2). In response to the need for a relatively simple interactive version, the Cholesky factorization $(C F)$ function was written in APL, a language uniquely suited to matrix manipulation.

The $\mathrm{CF}$ monadic defined function is based on an algorithm presented in Timm (1975). ${ }^{1}$ Briefly, following Timm, triangular factorization of any gramian matrix (say A) is accomplished through the systematic formation of diagonal elements of a triangular factor matrix (say $\mathrm{T}^{\prime}$ ) in which, for the first row of $\mathrm{T}^{\prime}$,

$$
t_{11}=\sqrt{a_{11}}
$$

and

$$
\mathrm{t}_{1 \mathrm{j}}=\mathrm{a}_{1 \mathrm{j}} \mathrm{j} / \mathrm{t}_{11}, \quad 1 \leqslant \mathrm{j} \leqslant \mathrm{n},
$$

and for all succeeding rows,

$$
\begin{array}{ll}
\mathrm{t}_{\mathrm{ij}}=0, & 1<\mathrm{j}<\mathrm{i} \leqslant \mathrm{n}, \\
\mathrm{t}_{\mathrm{ii}}=\sqrt{\mathrm{a}_{\mathrm{ii}}-\sum_{\mathrm{k}=1}^{\mathrm{i}-1} \mathrm{t}^{2} \mathbf{k i}_{\mathrm{i}},} & 1<\mathrm{i} \leqslant \mathrm{n},
\end{array}
$$

and

$$
\mathrm{t}_{\mathrm{ij}}=\frac{\mathrm{a}_{\mathrm{ij}}-\sum_{\mathrm{k}=1}^{\mathrm{i}-\mathrm{I}^{1}} \mathrm{t}_{\mathrm{ki}} \mathrm{t}_{\mathrm{kj}}}{\mathrm{t}_{\mathrm{ii}}}, \quad 1<\mathrm{i}<\mathrm{j} \leqslant \mathrm{n},
$$

The author is indebted to N. H. Timm. Much, if not most, of this discussion follows directly from his excellent exegesis of matrix operations as applied to multivariate statistical analy sis. Thanks are also extended to Julie Johnson McGowan for her invaluable advice and assistance and to W. Lloyd Milligan for his critical reading of the manuscript. so that the upper triangular matrix $T^{\prime}$ is defined where

$$
\mathrm{A}=\mathrm{TT}^{\prime} \text {. }
$$

During the factorization, if any $\mathbf{t}_{\mathbf{i i}}=0$ whereby $\mathrm{A}$ would be less than full rank (i.e., PSD), then the ith row is replaced with zeros and the factorization is carried to completion.

Function Description and Use. To use the APL CF function presented in Figure 1, enter the name of the function (CF), followed by the APL variable identifying the matrix to be factored (right argument of CF). CF's assignable output is returned explicitly. Functions structured in this way enable the user to (1) display results without intervention, (2) assign output to a variable,

Table 1

Function CF: Use and Output

Example 1: (a) Cholesky Factorization, Symmetrical PD Matrix ; (b) Explicit Result

A1

$\begin{array}{rrrr}81 & 9 & 27 & 36 \\ 9 & 26 & 53 & 34 \\ 27 & 53 & 113 & 86 \\ 36 & 34 & 86 & 165\end{array}$

\begin{tabular}{|c|c|c|c|}
\hline 8 & 9 & 27 & 36 \\
\hline & 1 & 3 & 4 \\
\hline 2 & 3 & 13 & 26 \\
\hline 3 & 4 & 26 & 129 \\
\hline \multicolumn{4}{|l|}{$\mathrm{T} \leftarrow \mathrm{CF} A 2$} \\
\hline \multicolumn{4}{|l|}{$\mathrm{T}$} \\
\hline & 1 & 3 & 4 \\
\hline & 0 & 0 & 0 \\
\hline & 0 & 2 & 7 \\
\hline & 0 & 0 & 8 \\
\hline
\end{tabular}

CF A1

$\begin{array}{llrl}9 & 1 & 3 & 4 \\ 0 & 5 & 10 & 6 \\ 0 & 0 & 2 & 7 \\ 0 & 0 & 0 & 8\end{array}$

Example 2: (a) Cholesky Factorization, Symmetrical PSD Matrix; (b) Variable Assignment A2

Example 3: (a) Calculation of the Determinant, Gramian Matrix; (b) Expression Use; (c) Assignment A3

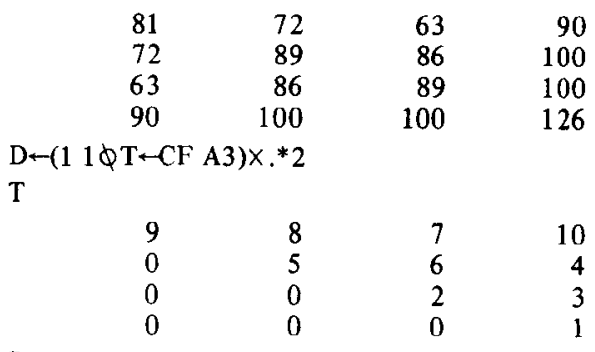

D 8100 


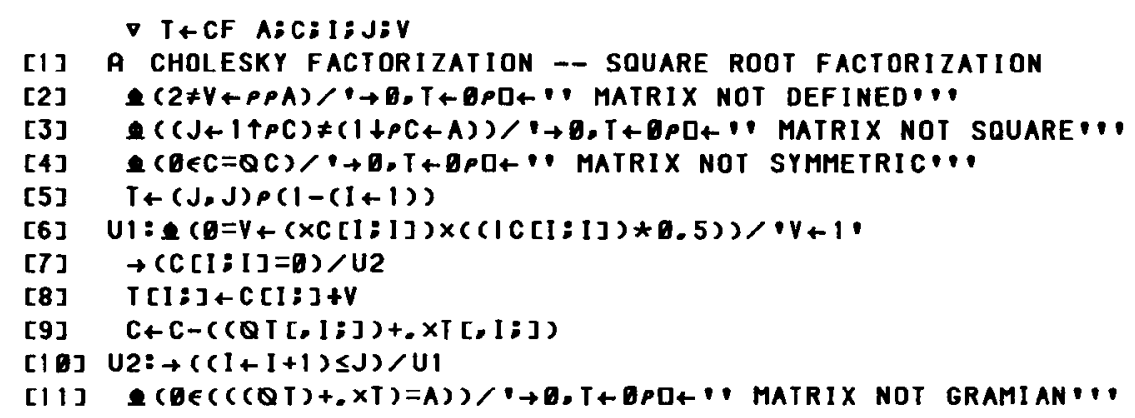

Figure 1. APL Function CF to compute the Cholesky factor of a gramian matrix. A Calcomp drum plotter (Model 1051) and a specially developed APL character set were used to produce Figure 1.

and, most important, (3) use the function in an expression. When returned, the output is either the upper right triangular factor or, given that the factor does not exist, a null or empty vector with an appropriate message, such as matrix not defined, matrix not square (rows $\neq$ columns), matrix not symmetric $\left(A \neq A^{\prime}\right)$, or matrix not gramian $\left(A \neq T T^{\prime}\right)$. For illustrations on the use of Function $\mathrm{CF}$, see examples in Table 1.

Particular attention should be paid to Example 3, Table 1, which shows one important use of CF (i.e., computing the determinant). The determinant (frequently used in multivariate analysis) is simply the squared product of the diagonal elements of any triangular factor (say $\mathrm{T}$ ):

$$
|A|=|T|^{2}=\prod_{i=1}^{n} t_{i i}^{2}
$$

Computer. Function CF was developed on an AMDAHL 470/V6-II under IBM's program product VS APL (Release 4.0) and was subsequently tested on an IBM 4341. All routines should be easily transported, with little or no modification, to other processors with an APL interpreter.

\section{REFERENCE NOTES}

1. Bock, R. D., \& Repp, B. H. ESL matrix operations subroutines for the IBM System/360 computers (Research Memorandum 11). Chicago: Statistical Laboratory, Department of Education, University of Chicago, August 1970.

2. Finn, J. D. MULTIVARIANCE: Univariate and multivariate analysis of variance, covarlance and regression. Ann Arbor, Mich: National Educational Resources, 1972.

\section{REFERENCES}

Finn, J. D. A general model for multivariate analysis. New York: Holt, Rinehart, \& Winston, 1974.

TıмM, N. H. Multivariate analysis with applications in education and psychology. Monterey, Calif: Brooks/Cole, 1975.

\section{NOTE}

1. When used, standard mathematical notation is employed. Uppercase letters denote a matrix (eg., A); its transpose is denoted by an affixed prime (e.g., $A^{\prime}$ ). A matrix element is denoted by the corresponding lowercase letter, with double subscripts indicating row and column, respectively (e.g., $a_{j j}$ ).

(Accepted for publication December 7, 1982.) 